\title{
Adiabatic switching for power dissipation reduction in complementary metal-oxide semiconductor (CMOS) gates of portable battery-powered devices
}

\author{
Ogunrinola O.B ${ }^{1}$, Oloketuyi S.I. ${ }^{1}$, Adegoke A.S ${ }^{2}$ \\ ${ }^{1}$ Department of Industrial Maintenance Engineering, Yaba College of Technology, Yaba, \\ Lagos State, Nigeria. \\ ${ }^{2}$ Department of Engineering, University of Leicester, Leicester LE1 7RH. United Kingdom.
}

\begin{abstract}
Electrical power dissipation in portable battery-powered devices has become worrisome to both consumers and producers of these devices. The need for this work is to investigate the practical low-power circuit solution for electrical power dissipation reduction in portable battery-powered devices. Experiments are performed on electrical power dissipation in Adiabatic switching and that of Complementary Metal-Oxide Semiconductor (CMOS) switching gates. The experiments are performed with the use of an electronic software simulator TINA TI ${ }^{M T}$ to simulate Adiabatic and CMOS switching circuits. The results obtain from simulated circuits are analyzed. The difference between electrical power dissipation in Adiabatic switching and CMOS switching reveals that Adiabatic switching reduces electrical power dissipation in portable battery-powered devices. This reduction in electrical power dissipation of battery-powered devices lowers the average electrical power consumption and lowers heat generation.
\end{abstract}

Keywords: Portable battery-powered Devices, Electrical power dissipation reduction, CMOS switching and Adiabatic switching

\section{INTRODUCTION}

In the last few years, there has been an increase in the demand for portable computation and communication devices. From portable telephones to sophisticated portable multimedia devices such as Personal Digital Assistants (PDAs) and notebook computers. The interest in portable battery-powered devices has brought the idea of developing a "switch" for power dissipation reduction in Complementary Metal-Oxide Semiconductor (CMOS) gates which is the main power consuming components in these devices. Power dissipation in this case, is that part of the electrical power taken by the device from the power supply which is converted into another form of energy (heat) which is not useful to the concerned device. However, it is a wasted power. Higher power dissipation implies more heat generation, which imposes higher cooling and packaging costs.

The reduction in power dissipation in CMOS gates will have multiple effects within the system such as reducing power consumption of the system, reducing the rate of recharging the battery and increasing the life span of the battery. Also, the reduction in power dissipation will improve the services obtained from these devices especially in environment where incessant failure in power supply is frequent. This means that once the battery has been fully charged, it will take longer period for it to discharge completely. In addition, since the electrical properties of the components that make up the devices are rated for specific temperature ranges, exceeding these ranges will alter the parameters and the behaviours of the circuits and this can cause a malfunction or reduction of the life span of the device.

Power dissipation in a CMOS circuit consists of the following components: dynamic (switching), shortcircuit and leakage powers. The switching component of the power occurs when energy is drawn from the supply to charge parasitic capacitors made up of gate, diffusion, and interconnect capacitance. In most cases, the switching component contributes more than $90 \%$ of the total power dissipation, making it the primary target for power reduction [1].

This energy dissipation by the switching component can be reduced by minimizing the circuit switching event, decreasing the node capacitance, reducing the voltage swing, or applying a combination of these methods. Yet, in all these cases, the energy drawn from the power supply is used only once before 
being dissipated. The comparable large power dissipation in conventional CMOS gates always lead to poor power efficiency. To increase the energy efficiency of logic circuits, other measures must be introduced for recycling the energy drawn from the power supply [2]. A class of logic circuits called adiabatic logic offers the possibility of further reducing the energy dissipated during switching events, by offering the possibility of recycling, or reusing some of the energy drawn from the power supply.

The load capacitance of an adiabatic switching circuit is charged by a constant current source. This circuit is similar to the equivalent circuit used to model the charge-up event in conventional CMOS circuits, with the exception that in conventional CMOS, the output capacitance is charged by a constant voltage source and not by a constant current source. A linear voltage ramp can be used instead of constant charging current. The constant-current charging of adiabatic switching process efficiently transfers energy from the power supply to the load capacitance. A portion of the energy thus stored in the capacitance can also be reclaimed by reversing the current source direction, allowing the charge to be transferred from the capacitance back into the supply [3] and [4]. This possibility is unique to adiabatic operation, since in conventional CMOS circuits the energy is dissipated after being used only once.

\section{CMOS and Adiabatic Conventional Circuits}

The simple circuit principle of the adiabatic amplifier can be extended to allow the implementation of arbitrary logic functions. Figures (1) and (2) show the general circuit topology of a conventional CMOS logic gate and an adiabatic counterpart.

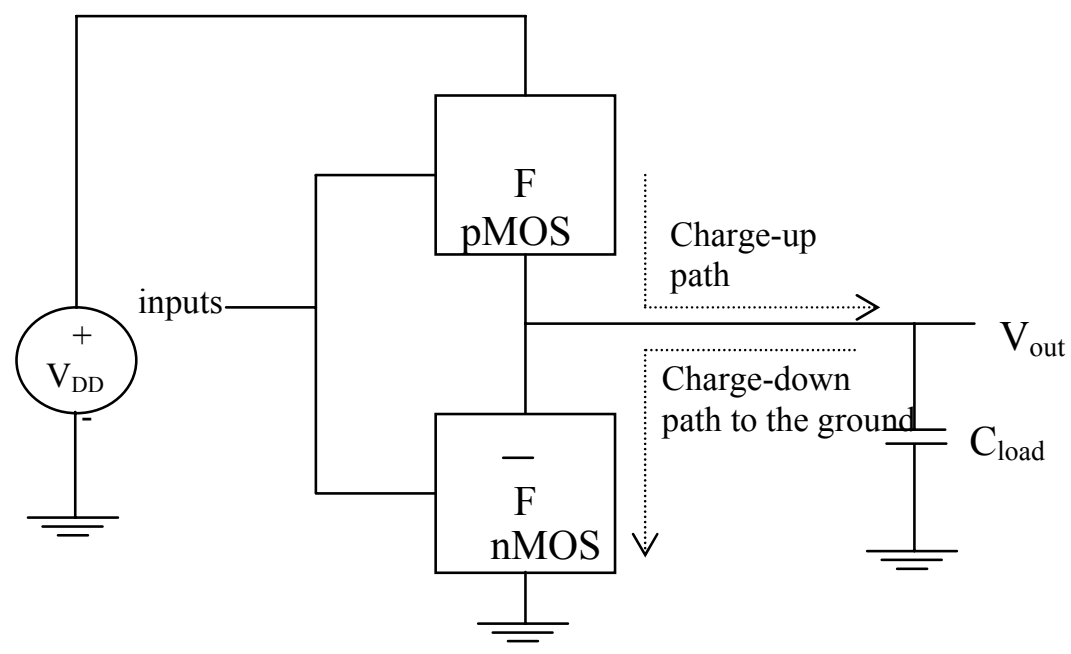

Fig 1 the general circuit topology of a conventional CMOS logic gate.

Switching power dissipation $\left(P_{\text {spd }}\right)$ of CMOS logic gate is denoted by:

$$
P_{\text {spd }}=f C V^{2} \text {, }
$$

where $f$ is the operating frequency, $C$ is the effective switching capacitance per cycle and $V$ is the voltage level of supply source. 
Am. J. Sci. Ind. Res., 2011, 2(4): 491-503

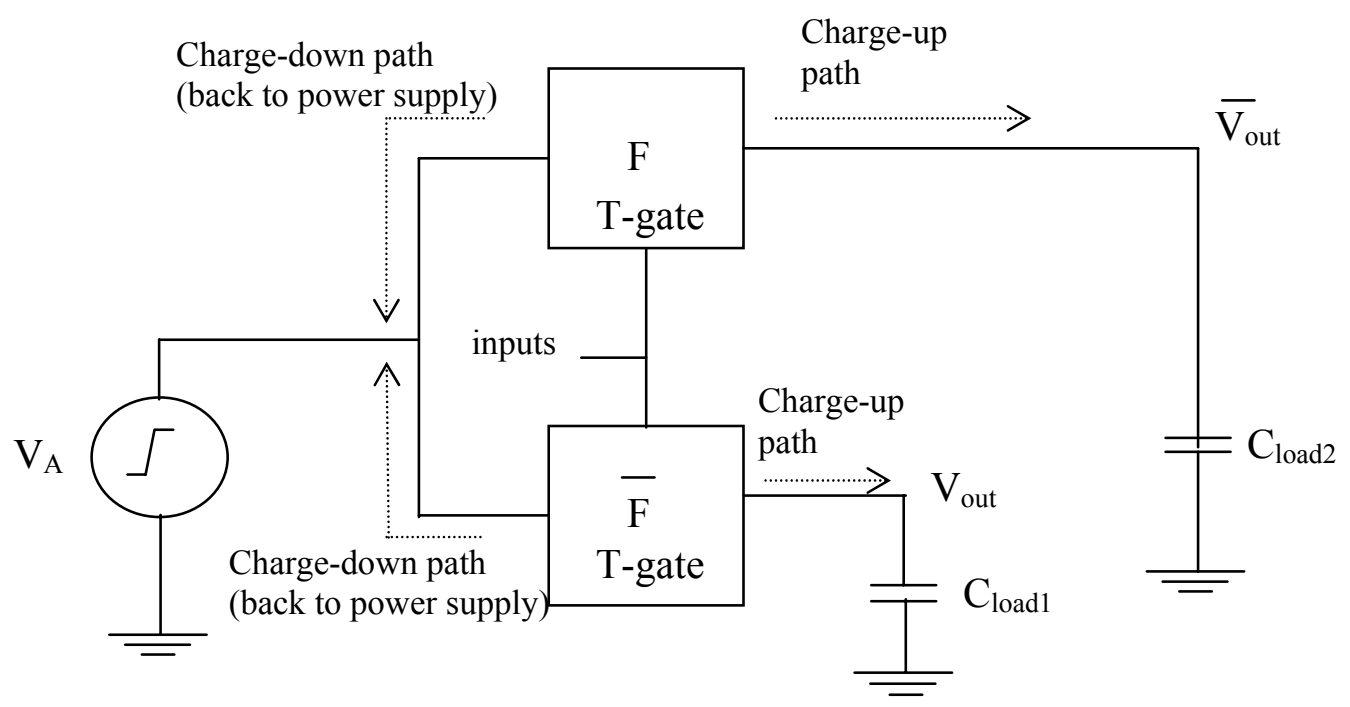

Fig 2 the general circuit topology of adiabatic logic circuit

Switching power dissipation ( $\left.E_{\text {diss }}\right)$ of Adiabatic switching circuit is denoted by:

$$
E_{\text {diss }}=\frac{R C}{T} C V_{c}^{2}(t) \text {. }
$$

This dissipated energy can be made arbitrarily small by increasing the charging time $\mathrm{T}$ of the supply and decreasing the on-resistance $R$ of the pMOS network, since energy dissipation $E_{\text {diss }}$ is inversely proportional to $T$ and directly proportional to $\mathrm{R}$.

To convert a conventional CMOS logic gate into an adiabatic gate, the pull-up and pull-down network must be replaced with complementary transmissiongate (T-gate) network. The T-gate network implementing the pull-up function is used to drive the true output of the adiabatic gate, while the T-gate network implementing the pull-down function drives the complementary output node. It should be noted that all inputs should be available in complementary form. The two networks in the adiabatic logic circuit are used to charge-up as well as charge-down the output capacitance, which ensures that the energy stored at the output node can be retrieved by the power supply (battery), at the end of each cycle. To allow adiabatic operation, the DC voltage source of the original circuit must be replaced by a pulsedpower supply with ramped voltage output. Actually, the circuit modifications which are necessary to convert a conventional CMOS logic circuit into an adiabatic logic circuit increase the device count by a factor of two. Again, the reduction in energy dissipation comes at the cost of slower switching speed, which is the ultimate trade-off in all adiabatic methods [5] and [6].

\section{MATERIALS AND METHOD}

TINA-TI ${ }^{\text {TM }}$ Simulations were performed to measure input and output powers of CMOS and Adiabatic switching circuits in order to calculate the power dissipation of each circuit with the same value of input voltage.

TINA-TI ${ }^{\mathrm{TM}}$ Simulator is a circuit design and simulation tool. It is ideal for designing, testing, and troubleshooting a broad variety of basic and advanced electronics circuits; including complex architectures. The tool is ideal for helping designers and engineers to develop and test circuit ideas. The TINA ${ }^{\mathrm{TM}}$ simulation software was used to simulate the circuits.

\section{RESULTS}

The circuits, simulated circuits, tables and the graphs of the results of the simulations were presented in the figures below. 
Am. J. Sci. Ind. Res., 2011, 2(4): 491-503

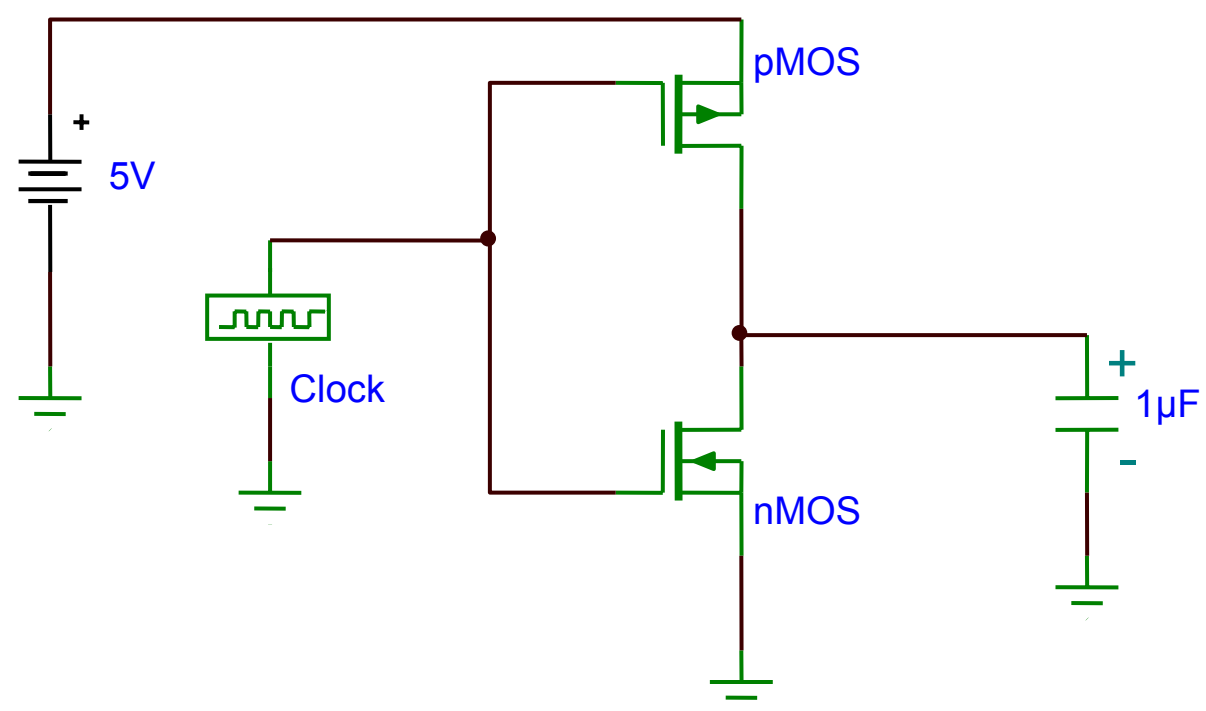

Fig 3 simulated CMOS switching circuit without Wattmeter CMOS switching circuit was simulated without Wattmeter to measure Input power and Output power

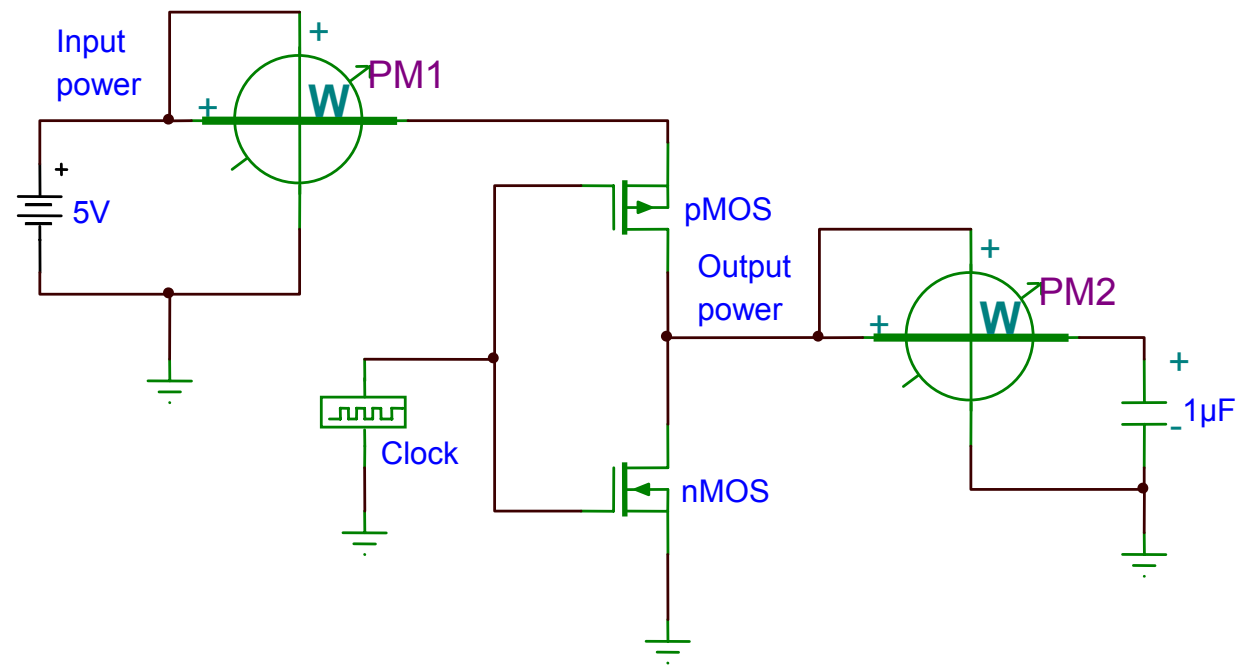

Fig. 4 simulated CMOS switching circuit with Wattmeter 
CMOS switching circuit was simulated with Wattmeter to measure Input power and Output power. The table of readings with graphs was shown bellow.

$\begin{array}{lrrr}\begin{array}{l}\text { Input voltage, in } \begin{array}{l}\text { Input power, P1 } \\ \text { ( V }) \\ \text { in (nW ) }\end{array} \\ 0\end{array} \quad \begin{array}{l}\text { Output power, P2 } \\ \text { in (nW ) }\end{array} & \begin{array}{l}\text { Dissipated power, } \\ \text { P1 - P2 in (nW ) }\end{array} \\ 0.5 & 11630 & 0 & 0 \\ 1.0 & 46080 & 0.0000396 & 11630 \\ 1.5 & 90390 & 0.000267 & 46080 \\ 2.0 & 142910 & 0.00222 & 90390 \\ 2.5 & 207370 & 0.00394 & 142910 \\ 3.0 & 284140 & 0.00614 & 207370 \\ 3.5 & 373550 & 0.00881 & 284140 \\ 4.0 & 475880 & 0.012 & 373550 \\ 4.5 & 591340 & 0.0156 & 475880 \\ 5.0 & 720130 & 0.0196 & 591340 \\ & & & 720130\end{array}$

Table 1 table of input power, output power and dissipated power against input voltage of CMOS circuit

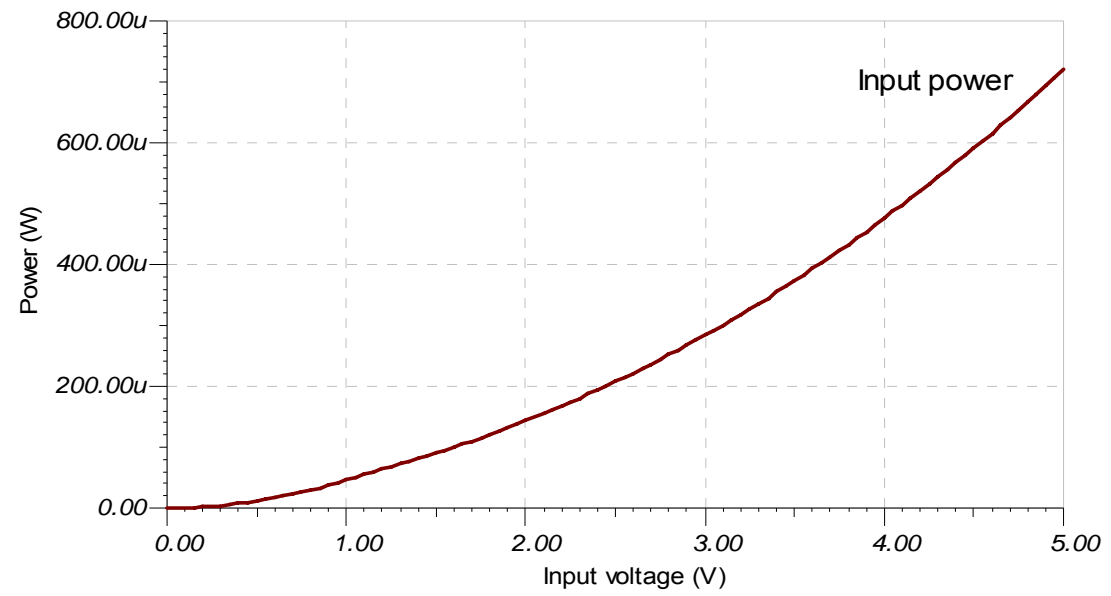

Fig 5 graph of input power against input voltage of CMOS circuit plotted by simulator. 
Am. J. Sci. Ind. Res., 2011, 2(4): 491-503

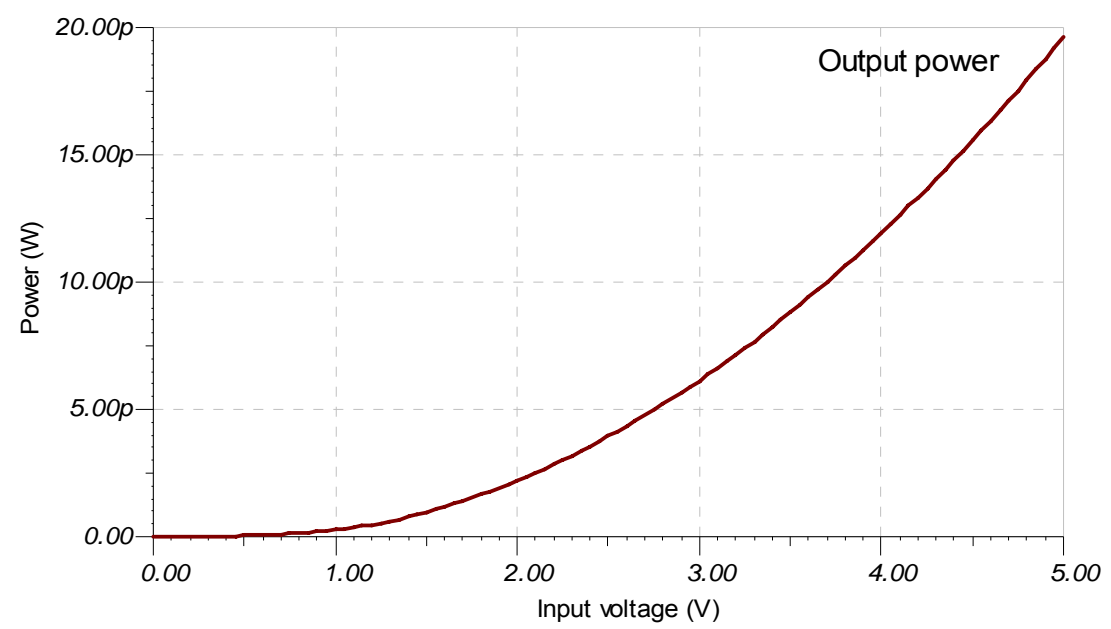

Fig 6 graph of output power against input voltage of CMOS circuit plotted by simulator.

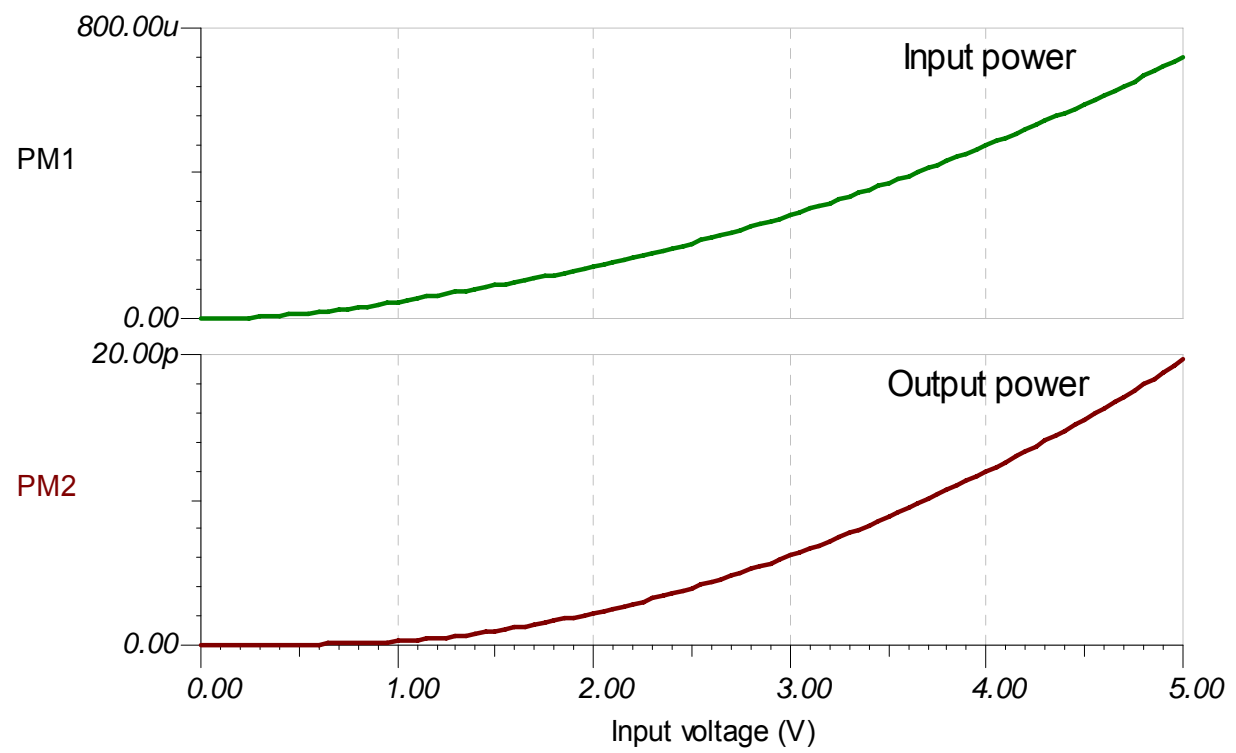

Fig 7 graphs of input power and output power against input voltage of CMOS circuit plotted by simulator. 
Am. J. Sci. Ind. Res., 2011, 2(4): 491-503

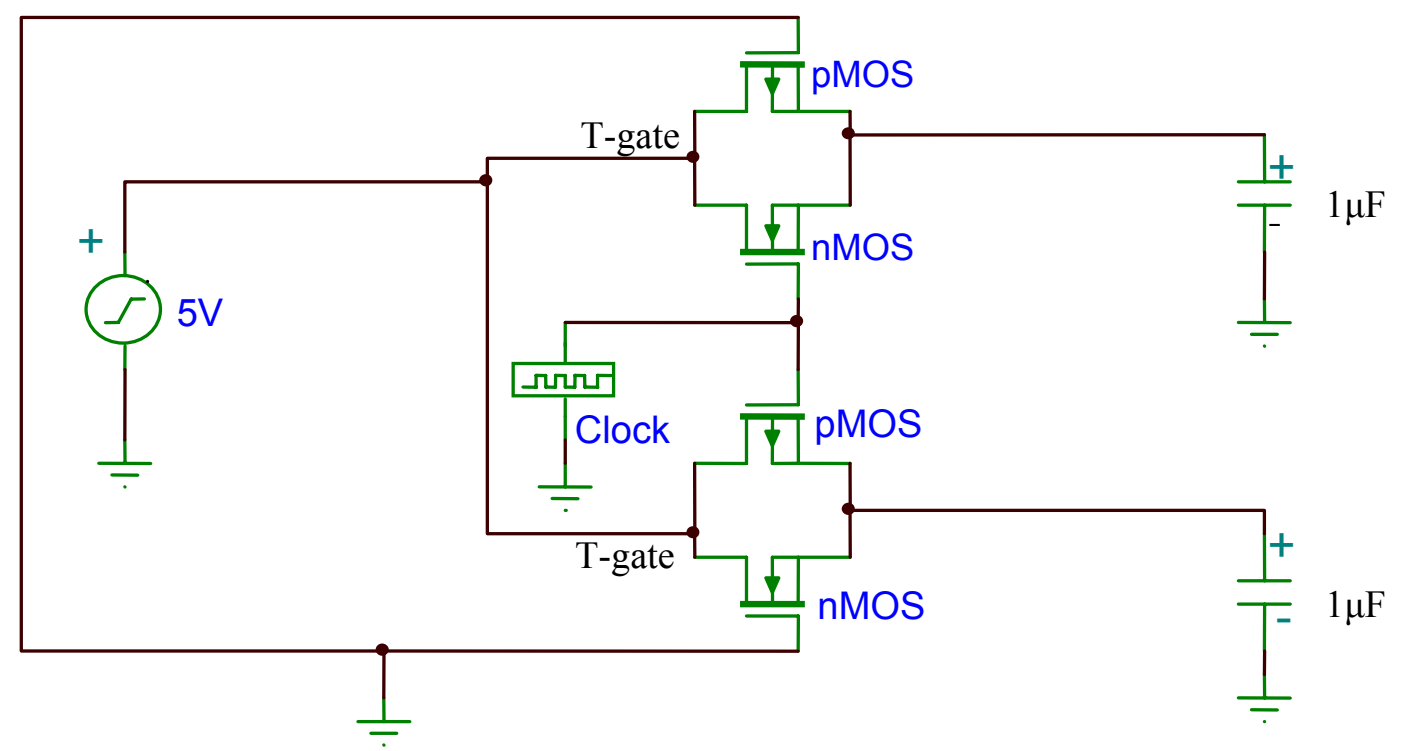

Fig 8 simulated adiabatic switching circuit without Wattmeter

Adiabatic switching circuit was simulated without Wattmeter to measure Input power and Output power.

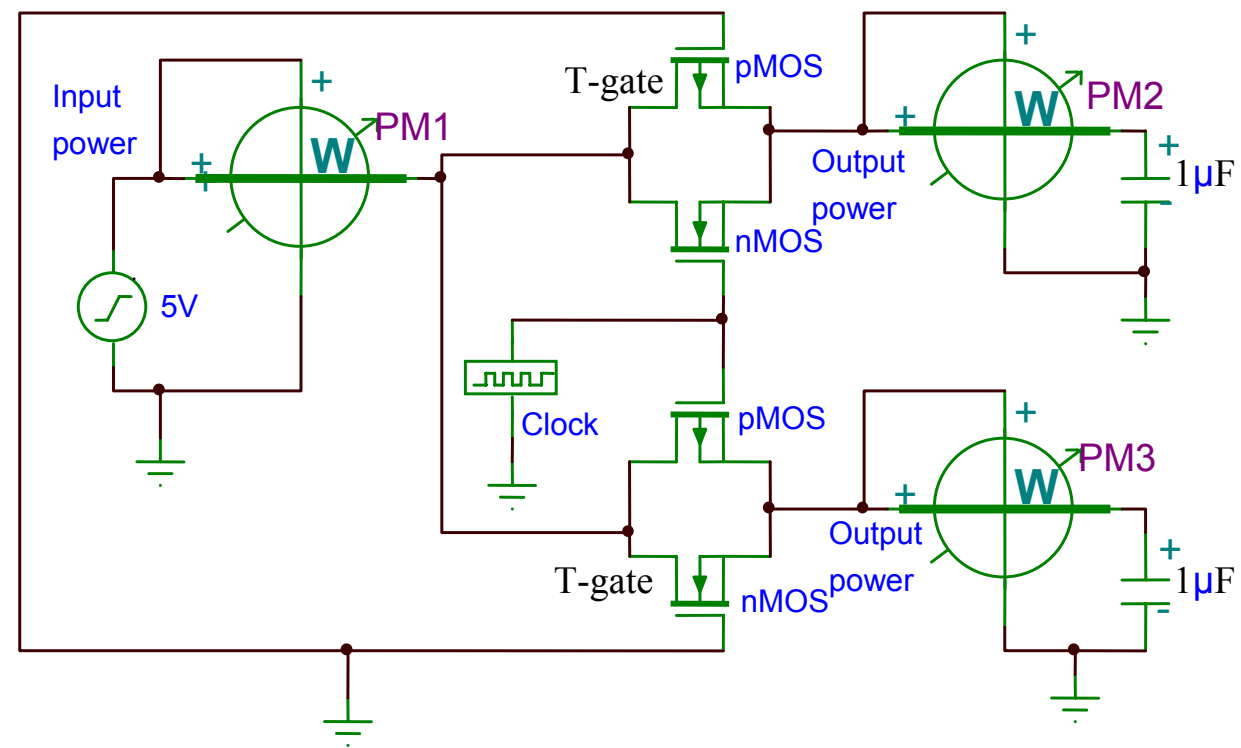

Fig 9 simulated adiabatic switching circuit with Wattmeter 
Am. J. Sci. Ind. Res., 2011, 2(4): 491-503

Adiabatic switching circuit was simulated with Wattmeter to measure Input power and Output power. The table of readings with graphs is shown bellow.

\begin{tabular}{|c|c|c|c|}
\hline Input voltage, & Input power, P1 & Output power, P2 & Dissipated power, \\
\hline$(\mathrm{V})$ & in $(n W)$ & in $(n W)$ & $P 1-P 2$ in $(n W)$ \\
\hline 0 & 0 & 0 & 0 \\
\hline 0.5 & 0.00325 & 0.00025 & 0.003 \\
\hline 1.0 & 0.013 & 0.001 & 0.012 \\
\hline 1.5 & 0.02926 & 0.00225 & 0.02701 \\
\hline 2.0 & 0.05202 & 0.004 & 0.04802 \\
\hline 2.5 & 0.08128 & 0.00625 & 0.07503 \\
\hline 3.0 & 0.11704 & 0.009 & 0.10804 \\
\hline 3.5 & 0.1593 & 0.01225 & 0.14705 \\
\hline 4.0 & 0.20806 & 0.016 & 0.19206 \\
\hline 4.5 & 0.26333 & 0.02025 & 0.24308 \\
\hline 5.0 & 0.3251 & 0.025 & 0.3001 \\
\hline
\end{tabular}

Table 2 table of input power, output power and dissipated power against input voltage of adiabatic circuit

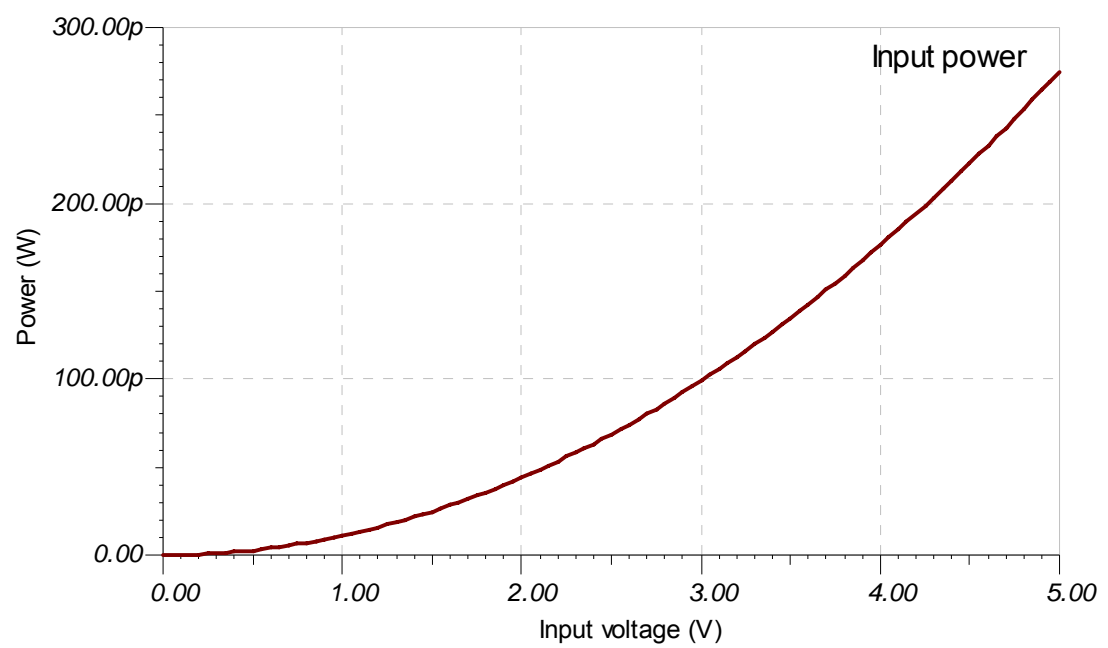

Fig 10 graph of input power against input voltage of adiabatic circuit plotted by simulator. 
Am. J. Sci. Ind. Res., 2011, 2(4): 491-503

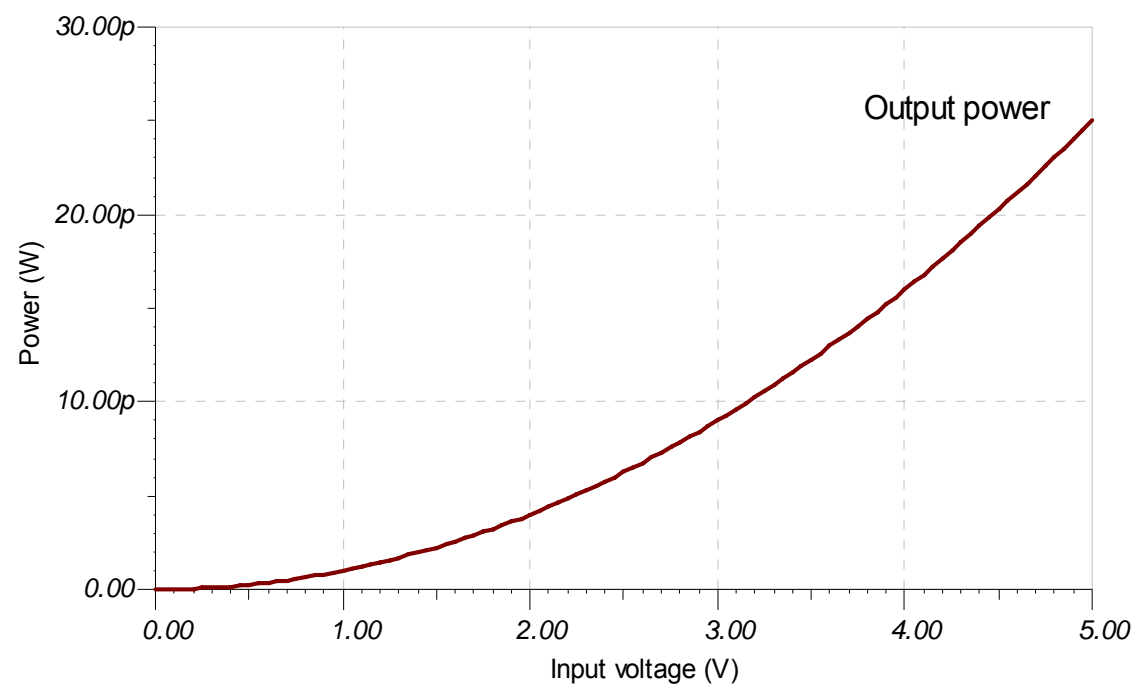

Fig 11 graph of output power against input voltage of adiabatic circuit plotted by simulator.

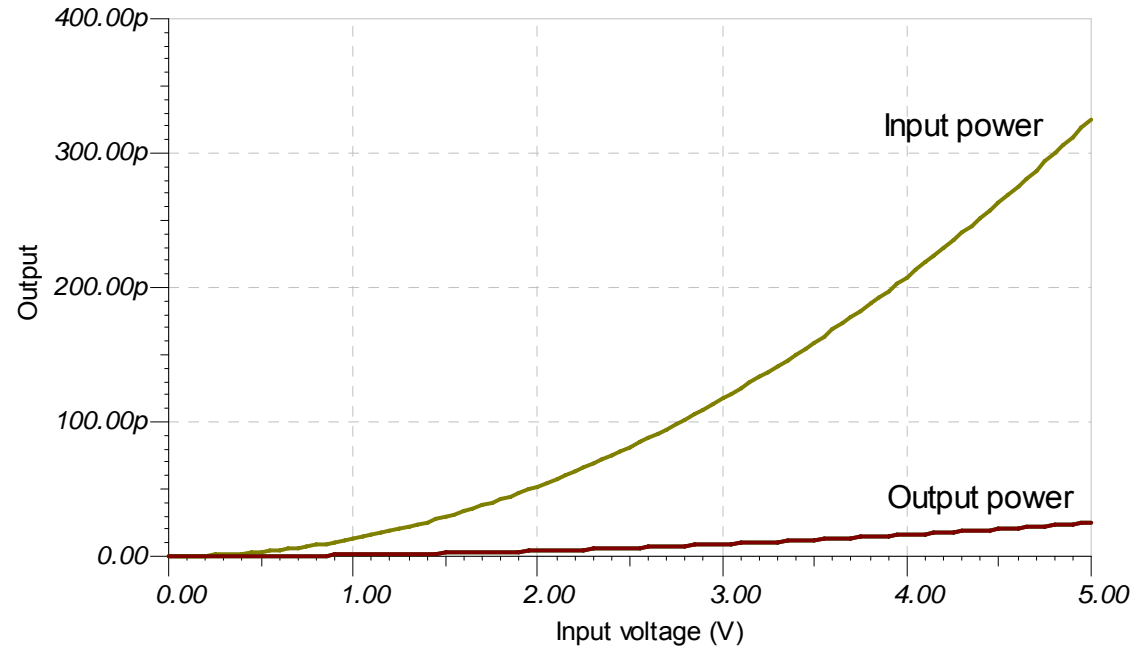

Fig 12 graphs of input power and output power against input voltage of adiabatic circuit plotted by simulator. 
Am. J. Sci. Ind. Res., 2011, 2(4): 491-503

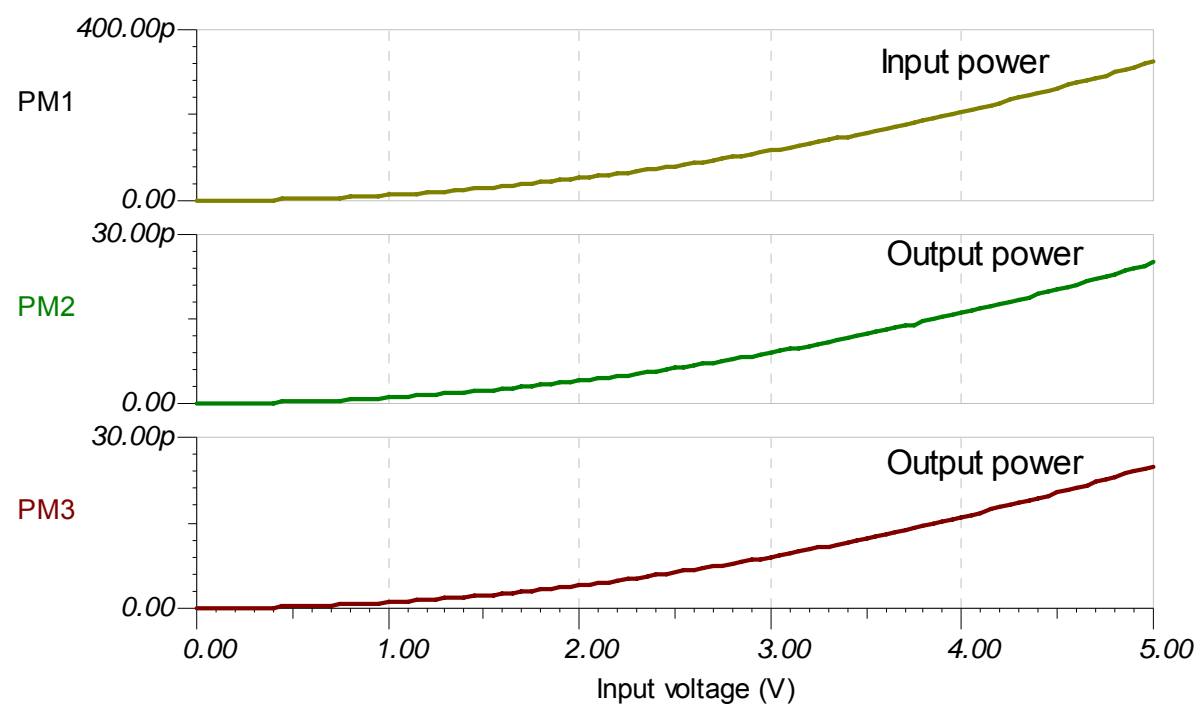

Fig 13 graphs of input power and output power against input voltage of adiabatic circuit plotted by simulator.

\begin{tabular}{|c|c|c|}
\hline Input voltage, & CMOS dissipated power, in ( $\mathrm{nW}$ ) & Adiabatic dissipated power, in ( $\mathrm{nW}$ ) \\
\hline (V) & i.e. Input power minus Output power & i.e. Input power minus Output power \\
\hline 0 & 0 & 0 \\
\hline 0.5 & 11630 & 0.003 \\
\hline 1.0 & 46080 & 0.012 \\
\hline 1.5 & 90390 & 0.02701 \\
\hline 2.0 & 142910 & 0.04802 \\
\hline 2.5 & 207370 & 0.07503 \\
\hline 3.0 & 284140 & 0.10804 \\
\hline 3.5 & 373550 & 0.14705 \\
\hline 4.0 & 475880 & 0.19206 \\
\hline 4.5 & 591340 & 0.24308 \\
\hline 5.0 & 720130 & 0.3001 \\
\hline
\end{tabular}

Table 3 table of CMOS dissipated power and adiabatic dissipated power against input voltage 
Am. J. Sci. Ind. Res., 2011, 2(4): 491-503

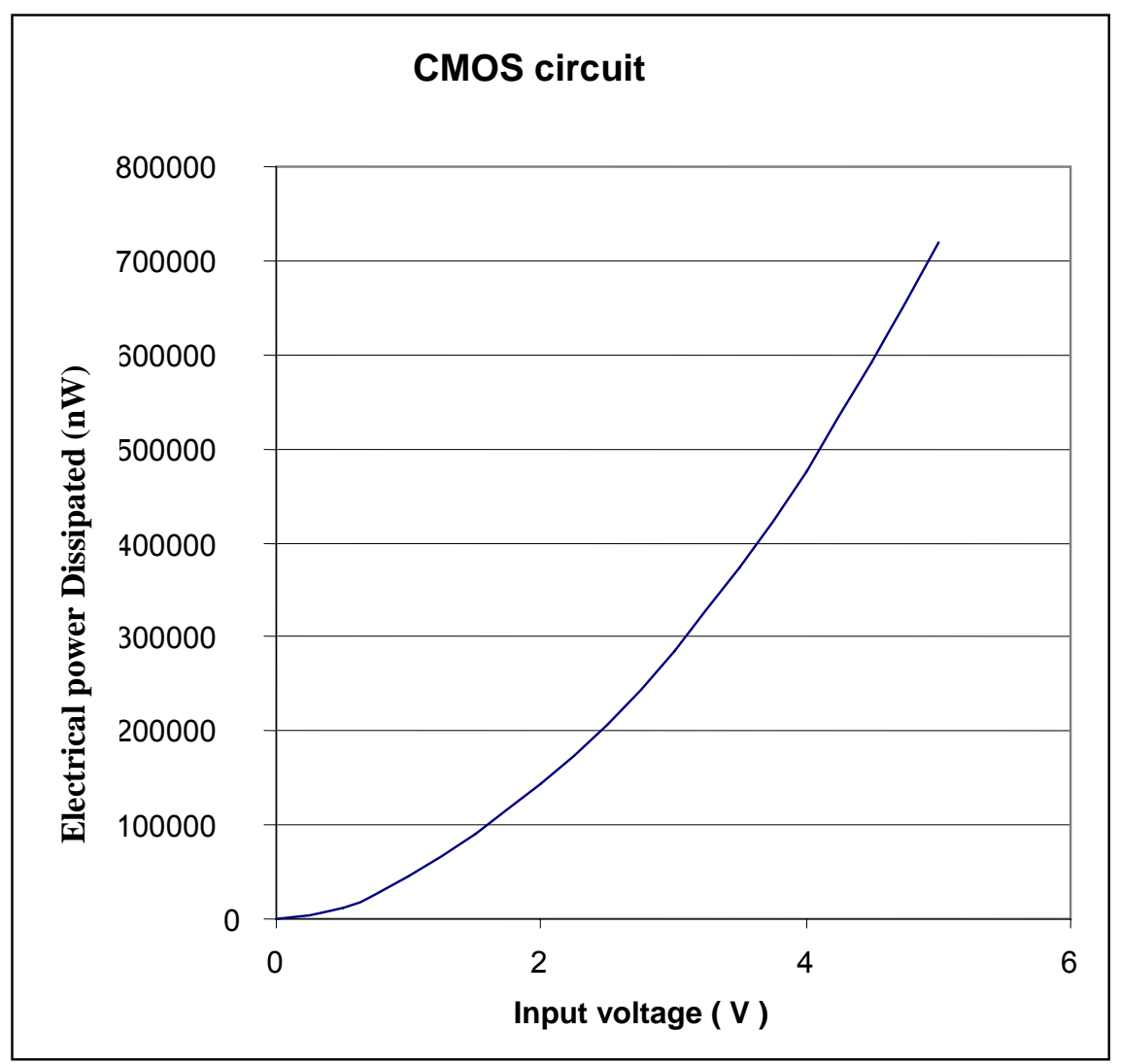

Fig 14 graph of dissipated power of CMOS circuit against input voltage plotted using spread sheet 
Am. J. Sci. Ind. Res., 2011, 2(4): 491-503

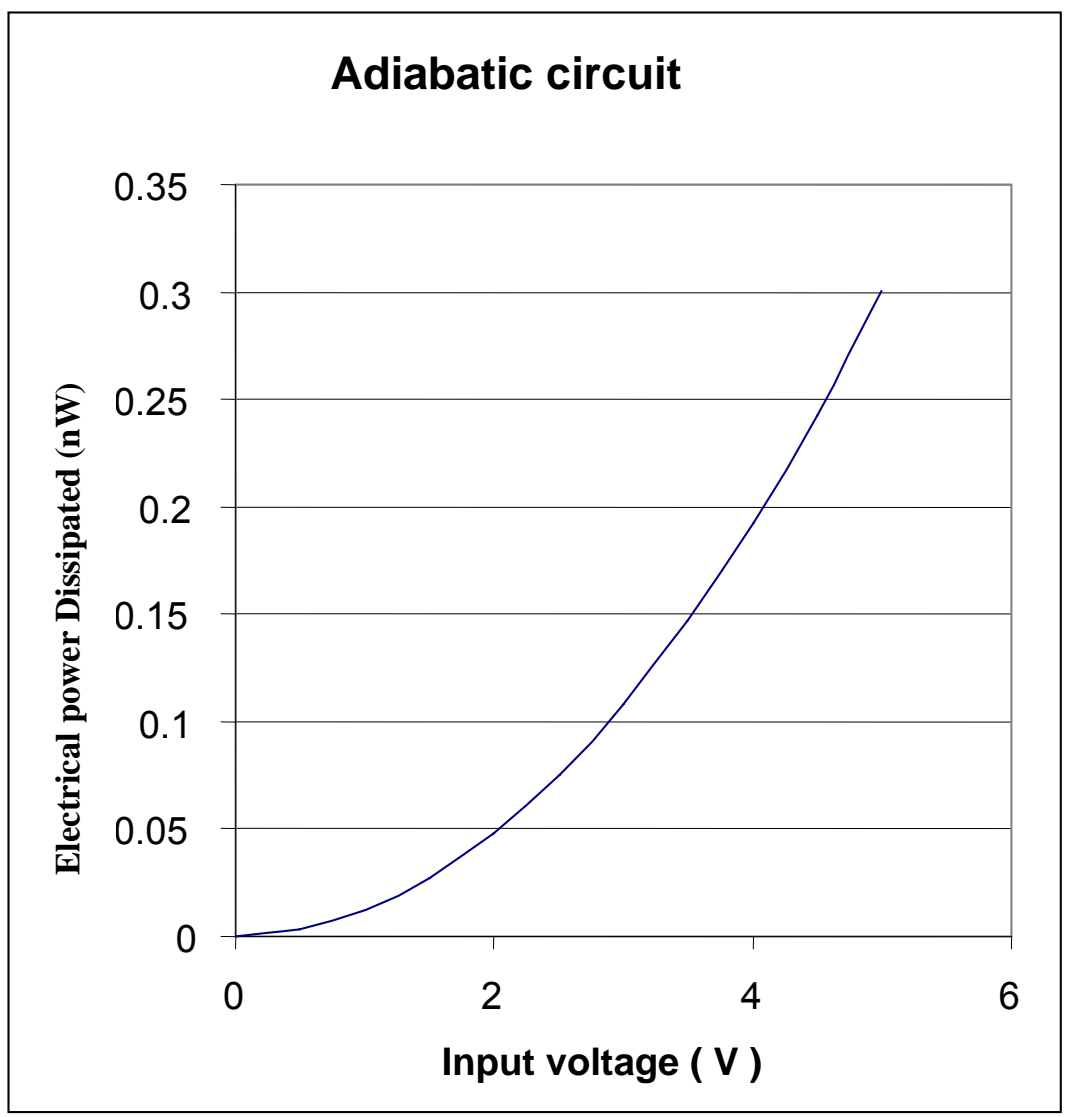

Fig 15 graph of dissipated power of adiabatic circuit against input voltage plotted by using spread sheet

\section{DISCUSSION}

Table 3, figure 14 and figure 15 above shown the summarized results of the experiments performed on CMOS and Adiabatic switching circuits, these results revealed that Adiabatic switching circuit dissipated very small electrical power as compared with CMOS switching circuit. Once the DC voltage source of CMOS switching circuit is replaced by a pulsedpower supply with ramped voltage output for Adiabatic switching circuit in potable battery-powered devices, electrical power dissipation of such devices will be drastically reduced. This high reduction in electrical power dissipation of Adiabatic switching of the device is due to the fact that part of the energy taken from the battery is re-used to charge (recycle) the battery again.

\section{CONCLUSION:}

Using adiabatic switching circuits in portable batterypowered devices will reduce electrical power dissipation, average electrical power consumption, heat generation and complexity of cooling system of the devices. Again, this will increase the period the battery serves the devices. This means, reducing electrical power dissipation of portable batterypowered devices, increasing the life span of both the batteries and the devices.

\section{RECOMMENDATION:}

With the astronomical increase in the demand for battery-powered portable computation and communication devices, adiabatic switching circuits should be adapted in the designs and fabrications of such devices so that the battery use in powering them can serve longer.

\section{REFERENCES}

1 A.P. Chandraksan and R.W. Brodersen, (1995). Low Power Digital CMOS Design, Norwell, MA: Kluwer Academic

Publishers http://www.epfl.ch/LSI2001/teaching/webcourse/c07/ch 07.html 
Am. J. Sci. Ind. Res., 2011, 2(4): 491-503

2 William C. Athas, Lars J. Svensson Low-Power Digital Systems Based on Adiabatic-Switching Principles.

http://www.cisi.columbia.edu/courses/spring2002/ee69 30/papers/003335009

3 Michael P. Frank, " Physical Limits of Computing Lecture \#24 Adiabatic CMOS” Spring 2002.

4 Roger L. Tokheim, (2004), Digital Electronics Principle and Applications Six Edition Published by Tata McGraw Hill Publishing Company Limited NEW DELHI
5 Ettore Amirante, Agnese Bargagli-Stoffi and Jurger Fischer, Varietion

of the Power Dissipation in Adiabatic Logic Gates.

http://www.patmos2001.eivd.ch/program/Repro\%5Cart-9-1

6 Vojin G. Oklobdzija, (2001), Computer Enginnering Handbook, CRC Press, Boca Raton London New York Washington 